\title{
Structure of Diglycerol Polyisostearate Nonionic Surfactant Micelles in Nonpolar Oil Hexadecane: A SAXS Study
}

\author{
Lok Kumar Shrestha', Rekha Goswami Shrestha', Keiichi Oyama², Makoto Matsuzawa² \\ and Kenji Aramaki ${ }^{*}$ \\ ${ }^{1}$ Graduate School of Environment and Information Sciences, Yokohama National University (Tokiwadai 79-7, Hodogaya-ku, Yokohama \\ 240-8501, JAPAN) \\ ${ }^{2}$ The Nisshin OilliO Group, Ltd.(1 Shinmori-cho, Isogo-ku, Yokohama 235-8558, JAPAN)
}

\begin{abstract}
Using a small-angle X-ray scattering technique, shape and size, and internal structure of diglycerol polyisostearate nonionic surfactant micelles in nonpolar oil $n$-hexadecane (HD) were investigated at $25^{\circ} \mathrm{C}$. Furthermore, the effect of added water on the structure of host reverse micelles was also investigated. The scattering data were evaluated by the generalized indirect Fourier transformation (GIFT) method and model fittings. It was found that diglycerol polyisostearate (abbreviated as (iso- $\left.\mathrm{C}_{18}\right)_{n} \mathrm{G}_{2}$, where $n=2-4$ represent the number of isostearate chain per surfactant molecule) spontaneously form reverse micelles in $\mathrm{HD}$ at $25^{\circ} \mathrm{C}$ and their geometry (shape and size, and internal structure) could flexibly be controlled by a small change in the lipophilic tail architecture of the surfactant, temperature, and water addition. Increasing number of isostearate chain per surfactant molecule decreases the micelles size favoring prolate-to-sphere type transition. This phenomenon could be best understood due to voluminous lipophilic part of the surfactant. Increasing temperature decreases the size of the reverse micelles due to enhanced interpenetration of the surfactant chain and the oil and also due to dominant hydrophobic character of the surfactant at higher temperatures. In the studies of effect of added water on the structure of micelles, it was found that the reverse micelles swell with water causing two dimensional micellar growths.
\end{abstract}

Key words: diglycerol polyisostearate, nonionic surfactants, reverse micelles, small-angle X-ray scattering (SAXS), generalized indirect Fourier transformation (GIFT), real-space pair distance distribution function

\section{INTRODUCTION}

Studies on reverse micelles formulation and their structural characterization have attracted a significant interest over the years because of its wide range of applications in several fields of chemistry. Reverse micelles stabilize reactive species that is insoluble in nonpolar solvents, and is also used as a size controlling micro-reactor for different aqueous chemical reactions ${ }^{1,2)}$. Reverse micelles have been used as template for the synthesis of nanomaterials for a long time ${ }^{3-9)}$. It has been found that the structure of the nanomaterials largely depends on the structure (size and shape) of the template micelles ${ }^{10)}$. Reverse micelles have an inverse structure in comparison to the conventional normal micelles in aqueous systems and are often called inverse or inverted micelles. In reverse micelles, water-soluble hydro- philic part of the amphiphile forms core and the lipophilic part of the amphiphile are in a direct contact with the oil phase. Due to an unfavorable direct contact between the hydrophilic part and oils, reverse micelles are usually formed when an amphiphile is added into nonpolar solvents. Reverse micelles are mostly observed in the ternary mixtures of surfactant/water/oil, mostly in oil rich regions ${ }^{11-13)}$. Furthermore, they have also been observed in aqueous systems of lipophilic surfactant in surfactant rich regions ${ }^{14,15)}$. Only few reports exist that describes the formation of reverse micelles in organic solvents without water addition ${ }^{16-20)}$.

The ternary mixtures of water/Aerosol OT(AOT)/oils or water/lecithin/oils were the mostly chosen systems for the studies of reverse micelles so far $^{21-26)}$. In the studies of non-

\footnotetext{
*Correspondence to: Kenji Aramaki, Graduate School of Environment and Information Sciences, Yokohama National University, Tokiwadai 79-7, Hodogaya-ku, Yokohama 240-8501, JAPAN

E-mail: aramakik@ynu.ac.jp

Accepted January 13, 2010 (received for review August 19, 2009)

Journal of Oleo Science ISSN 1345-8957 print / ISSN 1347-3352 online

http://www.jstage.jst.go.jp/browse/jos/
} 
aqueous phase behavior of mono-and diglycerol fatty acid esters in a variety of organic solvents we have found evidences on the formation of various self-assembled structures in the latter systems ${ }^{19)}$. Although liquid crystalline phases were absent in the temperature-composition diagrams of the monoglycerol fatty acid ester/oil systems, an isotropic solution of micellar aggregates was observed at elevated temperatures ${ }^{27)}$. In the diglycerol based surfactant/oil systems, we have found swollen lamellar liquid $\operatorname{crystal}\left(\mathrm{L}_{\alpha}\right)$ phase in the surfactant axis, and a dispersion of reverse vesicles in the solvent axis at $25^{\circ} \mathrm{C}$. The $\mathrm{L}_{\alpha}$ phase transform into an isotropic solution phase consisting of reverse micelles upon heating. It has been found that diglycerol monolaurate, and monomyristate would best offer a variety of reverse micellar structures such as globular, prolate, short rods, cylinder and planar depending on the solvent properties, temperature, composition and added wa$\operatorname{ter}^{28,29)}$. These mono-and diglycerol based nonionic surfactants form reverse micelles only at higher temperature and, hence, limit their practical application. Recently, we have formulated diglycerol polyisostearate-based reverse micelles in different organic oils at normal room temperature. It was found that the structure of micelles largely depends on the solvent properties, temperature, and water addition $^{30)}$.

The paper at hand is an extension of our previous study on the formulation of reverse micelles with a long chain lipophilic glycerol based nonionic surfactants at room temperature. Here, we present structure of diglycerol polyisostearate (abbreviated as $\left(\text { iso- } \mathrm{C}_{18}\right)_{n} \mathrm{G}_{2}, n=2-4$ ) reverse micelles in nonpolar oil $n$-hexadecane (HD) at $25^{\circ} \mathrm{C}$. Effect of lipophilic tail architecture of the surfactant, composition, temperature, and added water on the micellar structure will be discussed. For the structural characterization of reverse micelles, a small-angle X-ray scattering (SAXS) technique has been used. The SAXS data were evaluated by inverse Fourier transformation utilizing indirect Fourier transformation (IFT) and generalized indirect Fourier transformation (GIFT) methods and by direct modeling.

\section{EXPERIMENTAL SECTION}

\subsection{Materials}

Diglycerol polyisostearate (abbreviated as (iso- $\left.\mathrm{C}_{18}\right)_{\mathrm{n}} \mathrm{G}_{2}, n$

$=1-4$ ) with purity $>99 \%$ were obtained from the Nisshin OilliO Group, Ltd., Yokohama, Japan. The surfactants were used without further purification. The nonpolar oil $n$-hexadecane (HD) with purity $99.5 \%$ was purchased from Tokyo Chemical Industry, Tokyo Japan. Millipore-filtered water was used to investigate the water effect. The schematic molecular structures of $\left(\right.$ iso- $\left.\mathrm{C}_{18}\right){ }_{n} \mathrm{G}_{2}(n=1-4)$ are given in Scheme 1.
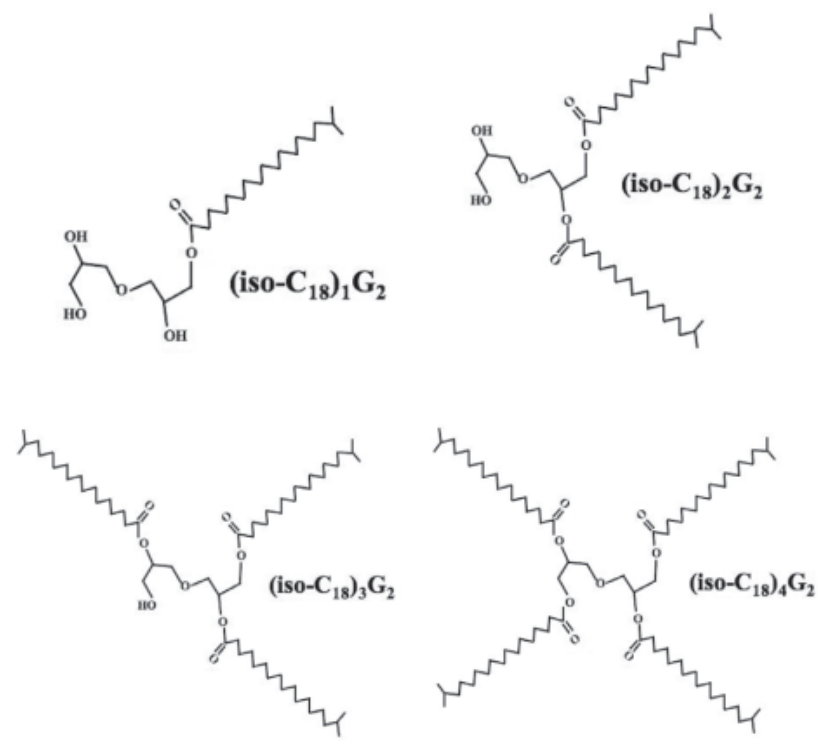

Scheme 1 Schematic Molecular Structures of Diglycerol Polyisostearates (iso- $\left.\mathrm{C}_{18}\right)_{\mathrm{n}} \mathrm{G}_{2}(n=1-4)$ Nonionic Surfactants.

\subsection{Methods}

2.2.1 Identification of equilibrium phases in the dilute regions

The equilibrium phases in the dilute region of the surfactant/oil binary mixture was identified by visual inspection through a crossed-polarizer. For this purpose, 5-25 wt\% of (iso- $\left.\mathrm{C}_{18}\right)_{\mathrm{n}} \mathrm{G}_{2},(n=1-4)$ /oil mixtures $(\sim 2 \mathrm{~g})$ were prepared in $\mathrm{HD}$ in clean and dry glass ampoules $(5 \mathrm{~mL})$ with the screw cap. The samples were mixed using dry thermobath, vortex mixer, and repeated centrifugation to achieve homogeneity. After mixing, the samples were kept in a temperature-controlled water bath at $25^{\circ} \mathrm{C}$ for $2 \mathrm{~h}$ to observe the equilibrium phases. An isotropic solution phase was observed in all the systems except the diglcyerol monoisostearate (iso- $\left.\mathrm{C}_{18}\right)_{1} \mathrm{G}_{2} /$ oil system, in which a turbid solution was observed. The turbid solution was stable with temperature up to $75^{\circ} \mathrm{C}$. Further temperature scans was not carried out. After mixing, the samples were placed in a water bath at $25^{\circ} \mathrm{C}$ for $2 \mathrm{~h}$ before SAXS measurements. The accuracy of the temperature reading in thermometer is \pm $0.5^{\circ} \mathrm{C}$. Water solubilization of the $10 \mathrm{wt} \%\left(\text { iso- } \mathrm{C}_{18}\right)_{2} \mathrm{G}_{2} / \mathrm{HD}$ system was determined first and the effect of added water on the reverse micellar structure was determined by SAXS measurements.

\subsubsection{Small-angle X-ray scattering (SAXS)}

SAXS measurements were carried out on a series of samples to investigate the effect of surfactant's tail size, composition, temperature, and added water to the reverse micellar structure. In the SAXS measurements, a SAXSess camera (Anton Paar, Austria) attached to a PW3830 sealedtube anode X-ray generator(PANalytical, Netherlands), 
which was operated at $40 \mathrm{kV}$ and $50 \mathrm{~mA}$ was used. An equipped Göbel mirror and a block collimator enabled us to obtain a focused monochromatic X-ray beam of $\mathrm{Cu}-\mathrm{K}_{\alpha}$ radi$\operatorname{ation}(\lambda=0.1542 \mathrm{~nm})$ with a well-defined line-shape. A thermostated sample holder unit(TCS 120, Anton Paar) was used to control the sample temperature.

The 2-D scattering pattern was recorded by an imagingplate (IP) detector (a Cyclone, Perkin Elmer, USA) and integrated into to one-dimensional scattered intensities, $I(q)$, as a function of the magnitude of the scattering vector $q=$ $(4 \pi / \lambda) \sin (\theta / 2)$ using SAXSQuant software (Anton Paar), where $\theta$ is the total scattering angle. All measured intensities were semi-automatically calibrated for transmission by normalizing a zero- $q$ attenuated primary intensity to unity, by taking advantage of a semi-transparent beam stop. All $I$ $(q)$ data were corrected for the background scattering from the capillary and the solvents, and the absolute scale calibration was made using water as a secondary standard.

Generalized indirect Fourier transformation (GIFT) method $^{31-34)}$ was used to obtain the real-space pair distance distribution function, $p(r)$, of the reverse micelles. This procedure relies on a basic equation of one-component globular particle systems, $I(q)=n P(q) S(q)$, and its extension to polydisperse systems, where $P(q)$ is the averaged form factor, $S(q)$ is the static structure factor, and $n$ is the number of particles in unit volume. As $p(r)$ is mathematically connected to $P(q)$ as

$$
P(q)=4 \pi \int_{0}^{\infty} p(r) \frac{\sin q r}{q r} d r
$$

an experimental $p(r)$ can be calculated as inverse Fourier transformation of $P(q)$. To suppress the influence of interparticle interference scattering on the evaluation of $p(r)$ that generally leads to highly underestimated maximum size of the scattering object, an interaction potential model for $S(q)$ was to be involved, where we chose the averaged structure factor model ${ }^{35,36}$ of hard-sphere and PercusYevick closure relation to solve Ornstein-Zernike equation. The detailed theoretical description on the method has been given elsewhere ${ }^{37-39)}$.

Indirect Fourier transformation (IFT) allows a separation into length and cross-section if the $p(r)$-function reveals cylindrical structure providing that the axial length at least three times longer than the cross-sectional diameter. Theoretically, the radial electron density profile, $\Delta \rho_{\mathrm{c}}(r)$, is connected to the cross-sectional pair-distance distribution function, $p_{c}(r)$, as ${ }^{40)}$

$$
p_{\mathrm{c}}(r)=r \Delta \tilde{\rho}_{\mathrm{c}}^{2}(r)
$$

Using IFT, the $p_{\mathrm{c}}(r)$ can directly be calculated from the experimental scattered intensity $I(q)$ via

$$
I(q) q=\pi L I_{\mathrm{c}}(q)=2 \pi^{2} L \int_{0}^{\infty} p_{\mathrm{c}}(r) J_{0}(q r) d r
$$

where $J_{0}(q r)$ is the zeroth-order Bessel function. The yielded $p_{\mathrm{c}}(r)$ can then be used to calculate $\Delta \rho_{\mathrm{c}}(r)$ by the deconvolution procedure $\mathrm{e}^{41,42)}$.

\subsubsection{Rheometry}

The rheological measurements were performed in a stress-controlled rheometer, AR-G2 (TA instrument) using cone-plate geometries (diameter $60 \mathrm{~mm}$ with a cone angle of $1^{\circ}$ ) with the plate temperature controlled by a peltier unit, which uses the peltier effect to rapidly and accurately control heating and cooling.

\section{RESULTS AND DISCUSSION}

\subsection{Isothermal phases in the dilute regions}

Equilibrium phases in the dilute regions of the diglycerol polyisostearate (iso- $\left.\mathrm{C}_{18}\right)_{\mathrm{n}} \mathrm{G}_{2},(n=1-4)$ /hexadecane (HD) systems were identified by visual inspection at $25^{\circ} \mathrm{C}$. It was found that in the dilute regions ( 5 to $25 \mathrm{wt} \%$ ) the surfactants $\left(\text { iso- } \mathrm{C}_{18}\right)_{n} \mathrm{G}_{2},(n=2-4)$ form an isotropic solution in HD at $25^{\circ} \mathrm{C}$. However, in the $\left(\text { iso- } \mathrm{C}_{18}\right)_{1} \mathrm{G}_{2} / \mathrm{HD}$ system, a turbid solution phase was observed. The turbid solution phase was present over a wide range of temperatures. Previous study has shown that the glycerol monoisostearate, (iso- $\left.\mathrm{C}_{18}\right)_{1} \mathrm{G}_{1}$, surfactant having only one glycerol molecule as a hydrophilic moiety forms an isotropic reverse micellar solution in $\mathrm{HD}$ at $25^{\circ} \mathrm{C}^{43)}$. Thus, the turbid solutions observed in the $\left(\text { iso- } \mathrm{C}_{18}\right)_{1} \mathrm{G}_{2} / \mathrm{HD}$ system can be attributed to the increased hydrophilicity of the surfactant. In the following sections, we will discuss the effect of nature of surfactant (mainly tail architecture), composition, and temperature on the structure of reverse micelles in the $\left(\right.$ (iso- $\left.\mathrm{C}_{18}\right)$ $\left.{ }_{n} \mathrm{G}_{2}, n=2-4\right) / H D$ systems. Furthermore, we will discuss the water induced microstructure transition in the $10 \mathrm{wt} \%$ (iso- $\left.\mathrm{C}_{18}\right)_{2} \mathrm{G}_{2} / \mathrm{HD}$ system.

\subsection{Effect of tail architecture on the reverse micellar structure}

Figure 1 shows the results of SAXS measurements $(I(q)$ and pair-distance distribution functions, $p(r)$, obtained from the GIFT evaluation of the scattering data) for the 5 wt $\%\left(\left(\text { iso- } \mathrm{C}_{18}\right)_{\mathrm{n}} \mathrm{G}_{2}, n=2-4\right) / \mathrm{HD}$ systems at $25^{\circ} \mathrm{C}$. As it can be seen in Fig. 1a, the scattering function largely depends on the number of isostearate chain per surfactant molecule, $n$. With increasing $n$, the forward scattering intensities (in the low- $q$ regions) are suppressed greatly. The low- $q$ scattering intensity corresponds to the higher-r side of the $p(r)$-function, and hence, higher forward scattering intensity with a higher low- $q$ slope indicates the system with larger particles. Thus without going to the $p(r)$-function, merely judging from the scattering behavior, one can see that the size of the reverse micelles decreases with increasing the number of isostearate chain per surfactant molecule. In the $p(r)$-function presented in Fig. 1b, one can see 

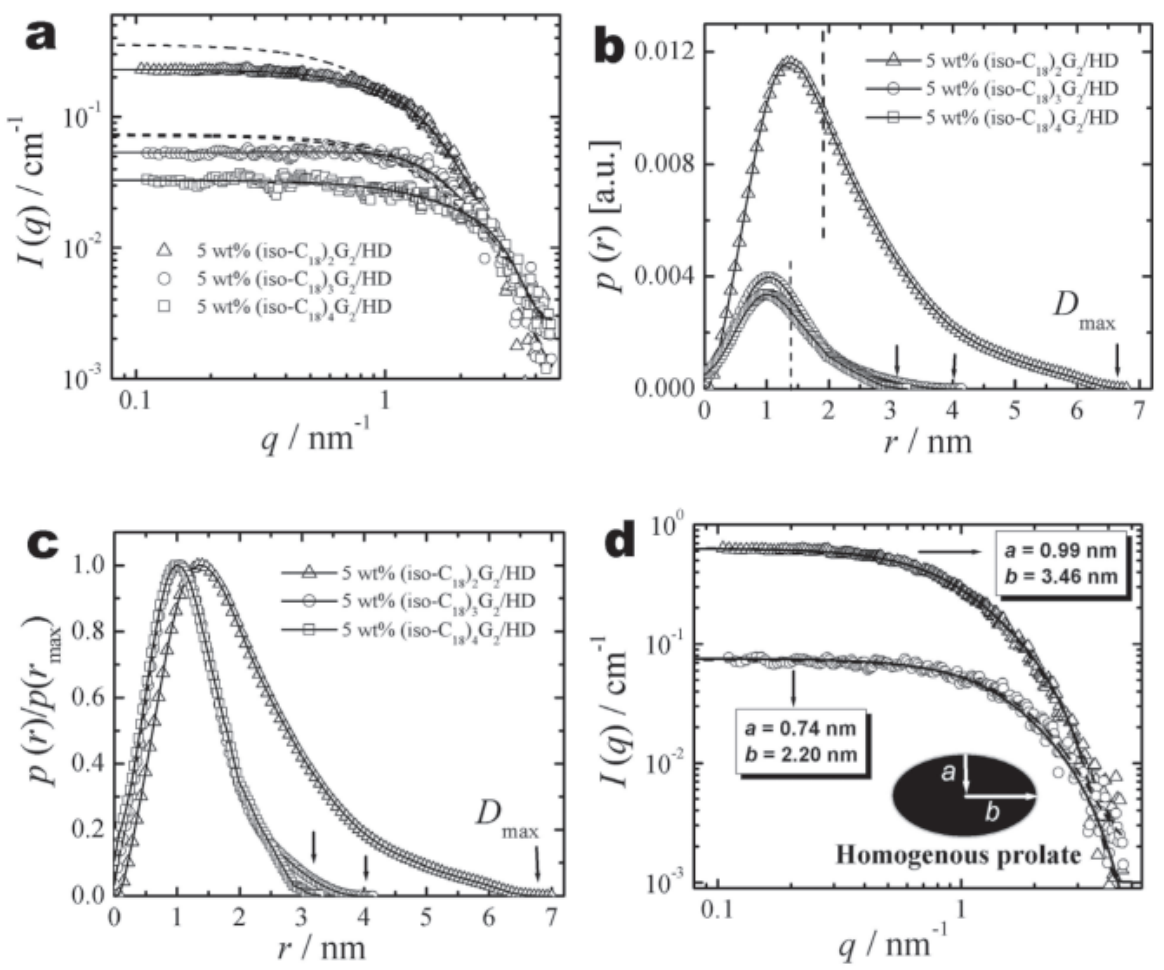

Fig. 1 (a) The Scattered Intensities, $I(q)$, for the $\left.5 \mathrm{wt} \%\left(\left(\text { iso- } \mathrm{C}_{18}\right)_{\mathrm{n}} \mathrm{G}_{2}, n=2-4\right)\right) /$ HD Binary Systems at $25^{\circ} \mathrm{C}$ in Absolute Scale, (b) the Pair-distance Distribution Functions, $p(r)$, (c) the Normalized $p(r)$-function $(p(r) /$ $p\left(r_{\max }\right)$ ), and (d) Model Fitting for Selected Systems (open triangle $=5 \mathrm{wt} \%$ $\left(\text { iso- } \mathrm{C}_{18}\right)_{2} \mathrm{G}_{2} / \mathrm{HD}$, and open circle $=5 \mathrm{wt} \%\left(\text { iso- } \mathrm{C}_{18}\right)_{3} \mathrm{G}_{2} / \mathrm{HD}$ ).

The solid and broken lines in panel a represent GIFT fit and the calculated form factor for $n$ particles existing in unit volume, $n P(q)$, respectively. The arrows and broken lines in panel $\mathrm{b}$ indicate the maximum dimension of the micellar core, $D_{\max }$, and the cross-sectional diameter of the reverse micellar core, respectively.

an asymmetric shape of the $p(r)$-curve in the $\left(\text { iso- } \mathrm{C}_{18}\right)_{2} \mathrm{G}_{2} /$ HD system with a pronounced peak in the low- $r$ side and an extended a downward convex shape tail in the higher- $r$ side. This confirms the ellipsoidal prolate type particles. However, upon changing surfactant from (iso- $\left.\mathrm{C}_{18}\right)_{2} \mathrm{G}_{2}$ to (iso- $\left.\mathrm{C}_{18}\right)_{4} \mathrm{G}_{2}$ via $\left(\right.$ iso- $\left.\mathrm{C}_{18}\right){ }_{3} \mathrm{G}_{2}$ the $p(r)$-curves becomes less asymmetric and also the maximum diameter of the micelles, $D_{\max }$, as indicated by down arrows decreases. It is interesting to point out that the micellar size is reduced by $\sim 52 \%$ upon changing surfactant from (iso- $\left.\mathrm{C}_{18}\right)_{2} \mathrm{G}_{2}$ to $\left(\text { iso- } \mathrm{C}_{18}\right)_{4} \mathrm{G}_{2}$. Moreover, the inflection point seen after the maximum in the $p(r)$-functions (indicated by broken line in Fig. 1b), shifts towards lower- $r$ side with increasing isostearate chain. This indicates that lipophilic tail architecture of the surfactant not only modifies the micellar size, but also the internal structure (cross-sectional diameter) of the reverse micelles. In Fig. 1c, we plot the normalized $p$ $(r)$-functions $\left(p(r) / p\left(r_{\max }\right)\right)$ for all the surfactant systems.
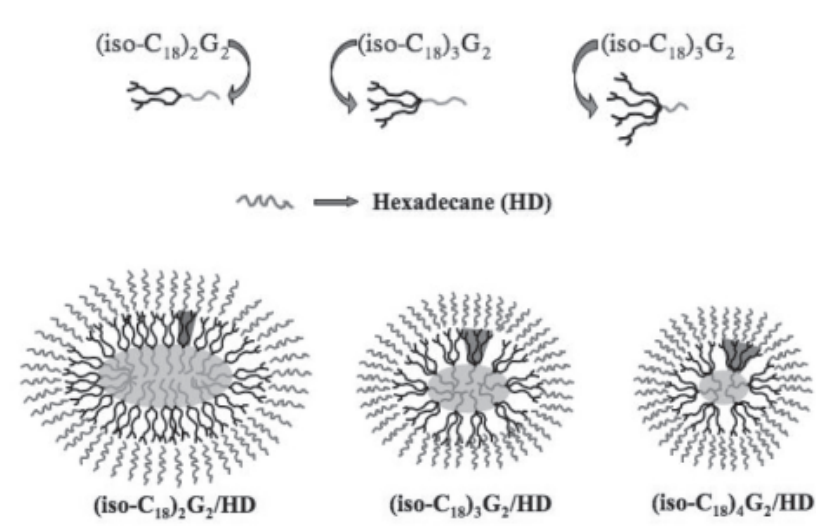

Scheme 2 Schematic Representation of Surfactant Molecules and Solvent (Top), and Cross-sectional View of the Diglycerol Polyisostearates (iso- $\left.\mathrm{C}_{18}\right)_{n} \mathrm{G}_{2}(n=2-4)$ Reverse Micelles in HD (bottom). 
The position of the maximum in the $p(r)$-function moves towards low- $r$ side with increasing the value of $n$ in (iso- $\mathrm{C}_{18}$ ) ${ }_{n} G_{2}$. This clearly shows that the micellar cross-section decreases with increasing $n$. Judging from the real space $p(r)$ -functions; one can anticipate the prolate-to-sphere type transition in the reverse micellar structure. The structure of the reverse micelles depending on the lipophilic tail architecture can be explained in terms of critical packing parameter, $c p p$. As we increase the number of isostearate chain per surfactant molecules, the $c p p$ increases due to voluminous surfactant chain and, hence, aggregates with more negative curvature are formed. The microstructure transition can best be seen in model shown in Scheme 2 .

Although the virtually model free inverse Fourier transformation technique (IFT or GIFT) is a very powerful tool for the structural characterization of colloidal particles such as micelles or microemulsions, the theoretical model fit based on different plausible models are still common in practice for the structural characterization colloidal dispersions, micelles, and microemulsions. Therefore, the results obtained from the GIFT method are complemented by the model fittings. In Fig. 1d, the solid and broken lines are the model and GIFT fit, respectively. The data were fitted using a model of a homogeneous prolate. The model fittings were carried out based on the method reported in detail elsewhere ${ }^{44)}$. As it can be seen from Fig.1d, the short and long axes of the ellipsoidal prolate, $a$ and $b$, obtained from the theoretical model fits are consistent with the results obtained from the GIFT method.

In Fig. 2, we present the steady state rheological behavior of the $\left.5 \mathrm{wt} \%\left(\left(\text { iso- } \mathrm{C}_{18}\right)_{\mathrm{n}} \mathrm{G}_{2}, n=2-4\right)\right) / \mathrm{HD}$ binary systems at $25^{\circ} \mathrm{C}$. All the systems follow Newtonian fluid like behavior and the viscosity increases with decreasing the value of $n$ in the (iso- $\left.\mathrm{C}_{18}\right)_{n} \mathrm{G}_{2}$, which is in a good agreement with the SAXS data. Namely, the decrease in the viscosity

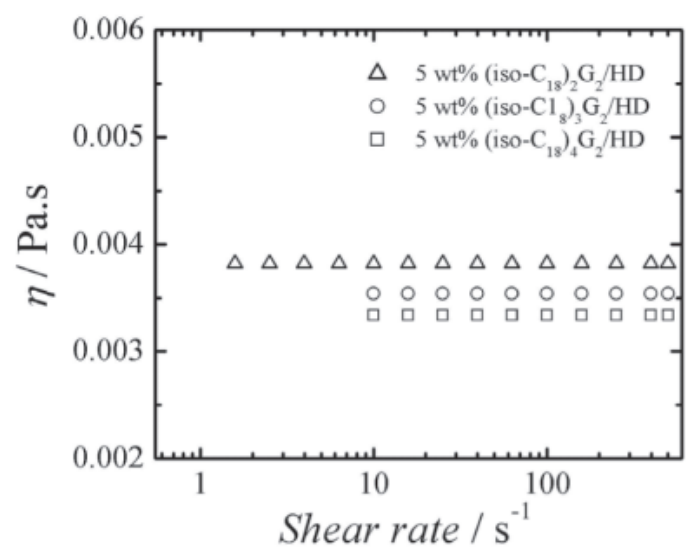

Fig. 2 The Steady Shear Rheology Data of 5 wt $\%$ (iso- $\left.\mathrm{C}_{18}\right)_{\mathrm{n}} \mathrm{G}_{2}, n=2-4 / \mathrm{HD}$ Systems at $25^{\circ} \mathrm{C}$. with increase in the number of isostearate chain per surfactant molecule is caused due to micelle shortening.

\subsection{Effect of surfactant concentration on the reverse micellar structure}

Effect of surfactant concentration on the micellar structure was investigated in $\left(\text { iso- } \mathrm{C}_{18}\right)_{2} \mathrm{G}_{2} / \mathrm{HD}$ system at $25^{\circ} \mathrm{C}$. The SAXS measurements were carried out on the (iso- $\mathrm{C}_{18}$ ) ${ }_{2} \mathrm{G}_{2} / \mathrm{HD}$ system over a wide range of concentration ( 5 to 20 wt \%) and the results are presented in Fig. 3.

As it can be seen in Fig. 3a, with increasing surfactant concentration from 5 to $10 \mathrm{wt} \%$, the scattering intensity increases throughout the $q$-range due to higher number density of the scattering particles in unit scattering volume. Minute observation of the scattering curves reveals that with further increasing concentration above $10 \mathrm{wt} \%$, the forward scattering intensity in the low- $q$ regions tends to suppress, and leads to formation of a weak, but growing interaction peak at intermediate $q$ values $\left(q \sim 1.5 \mathrm{~nm}^{-1}\right)$. This growing peak indicates the strong repulsive intermicellar interaction in the system at higher surfactant concentrations $^{45,46)}$.

As mentioned earlier, we have used GIFT method to evaluate our SAXS data, and thus it was possible to calculate the structure factor and form factor simultaneously without considering any form factor model. The structure factor profiles presented in Fig. $3 d$ indicate the presence of considerable intermicellar interactions, which are responsible for the growing peaks at $q \sim 1.5 \mathrm{~nm}^{-1}$ in the Fig. 3a. Minute observations of the $S(q)$-curves reveals that the structure factor peak, which reflects the mean distance between the micelles $\left(d=2 \pi / q_{\max }\right)$, tends to shift towards higher- $q$ side with increasing surfactant concentration indicating the decreasing mean distance between the neighboring micelles. Thus naturally, one can anticipate the higher intermicellar interaction at higher surfactant concentration. The decreasing $S(q=0)$ value with increasing surfactant concentration is the contribution of the reduced osmotic compressibility of the system. At higher- $q$ values, all the curves converge to unity, since at length scales smaller than the dimensions of the scattering entities interparticle interactions are not relevant. The actual structure factor peaks might differ from what is predicted for monodisperse hard spheres ${ }^{47)}$. Apart from polydispersity effects, some differences could arise due to a pair interaction softer than that predicted by the used hard-sphere model ${ }^{48)}$. Nevertheless, the polydispersity derived from the GIFT fit was low $<0.07$ despite the elongated micellar systems.

The shape and size of the micelles depend on the nature of the surfactants. Surfactant with critical packing parameter, $c p p,>1$ forms spheroid type micelles in nonaqueous systems. With increasing surfactant concentration above critical micelle concentration $(\mathrm{cmc})$, the number density of micelles increases and, hence, the intermicellar forces 

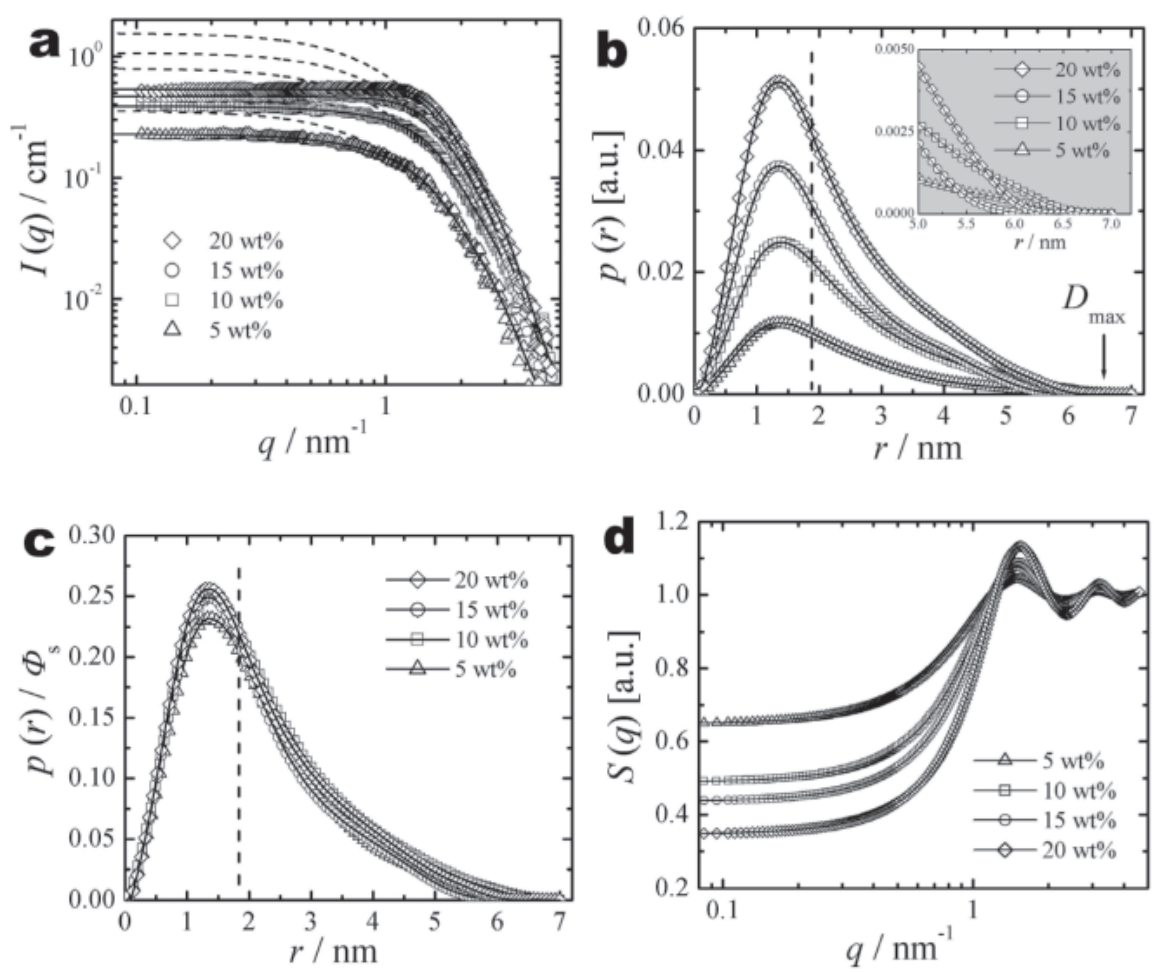

Fig. 3 (a) The Scattering Intensities, $I(q)$, of the (iso- $\left.\mathrm{C}_{18}\right)_{2} \mathrm{G}_{2} / \mathrm{HD}$ System at Different Surfactant Concentrations; 5, 10, 15, and $20 \mathrm{wt} \%$ in Absolute Unit at $25{ }^{\circ} \mathrm{C}$, (b) the Corresponding Real-space $p(r)$-Functions, (c) the Normalized $p(r)$ Functions $\left(p(r) / \phi_{\mathrm{s}}\right)$, and (d) the Structure Factor Curves.

The solid and broken lines in panel a represent GIFT fit and the calculated form factor for $n$ particles existing in unit volume, $n P(q)$, respectively. The arrow and broken line after the maximum of the $p(r)$ function in panel $\mathrm{b}$ highlight the maximum dimension, and the crosssection diameter of the micellar core, respectively.

come into play, which work perpendicular to the interface and are repulsive in nature. The repulsive intermicellar interactions induce micelle ordering at a particular surfactant volume fraction with the symmetry depending on the micellar shape. In the present system, we have found a clear signature of the formation of slightly elongated ellipsoidal prolate type micelles in the $\left(\text { iso- } \mathrm{C}_{18}\right)_{2} \mathrm{G}_{2} / \mathrm{HD}$ system judging from the pronounced peak in the low- $r$ side and an extended, but downward convex tail in the higher- $r$ side of the $p(r)$-curves. Changing surfactant concentration could not change the shape and size of micelles. The maximum dimension of the micelles, $D_{\max }$, remains apparently the same regardless of a wide variation in the surfactant concentration from 5 to $20 \mathrm{wt} \%$ see Fig. 3b. Furthermore, the inflection point seen on the higher- $r$ side of the maximum of $p(r)$, as highlighted by a dotted line at $r \sim 1.95 \mathrm{~nm}$ in Fig. $3 \mathrm{~b}$ are virtually unchanged indicating that the crosssectional structure is unaffected by the concentration variation. Figure $3 c$ shows the normalized curves by the sur- factant volume fraction $\Phi_{\mathrm{s}}$. All the curves lie on top of each other. If there would a micellar growth, the curves height would increase with the volume fraction of the surfac$\operatorname{tant}^{49)}$.

As it was mentioned in the experimental section, a separation into length and cross-section is possible if the $p(r)$ -curve reveals elongated or cylindrical structure providing that the axial length is at least three times longer than the cross-section. The cross-section diameter of the (iso- $\mathrm{C}_{18}$ ) ${ }_{2} \mathrm{G}_{2} / \mathrm{HD}$ system is estimated to $\sim 1.95 \mathrm{~nm}$ from the inflection point of the $p(r)$-function after the maximum (Fig. 3b). To quantify this parameter, we have performed direct crosssection analysis by using indirect Fourier transformation (IFT) and calculated the cross-sectional pair-distance distribution function, $p_{\mathrm{c}}(r)$, from the experimental scattered intensity $I(q)$ via Eq. 3. The scattering length density profile, $\Delta \rho_{\mathrm{c}}(r)$, was then calculated by the deconvolution of the $p_{c}(r)^{41,42)}$. Figure 4 shows the cross-sectional pairdistance distribution functions, $p_{\mathrm{c}}(r)$, and the correspond- 

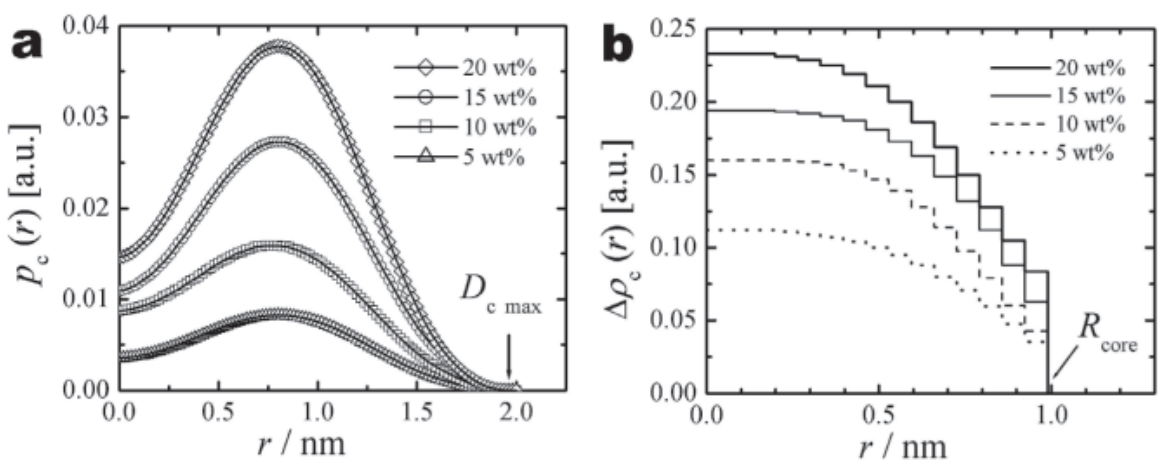

Fig. 4 (a) The Cross-sectional Pair-distance Distribution Function, $p_{\mathrm{c}}(r)$, for the (iso- $\left.\mathrm{C}_{18}\right)_{2} \mathrm{G}_{2} / \mathrm{HD}$ Systems at Different Surfactant Concentrations (5-20 wt $\%$ ) at $25^{\circ} \mathrm{C}$, and (b) the Corresponding Cross-section Radial Electron Density Profile, $\Delta \rho_{\mathrm{c}}(r)$, Calculated from the Deconvolution of the $p_{\mathrm{c}}(r)$
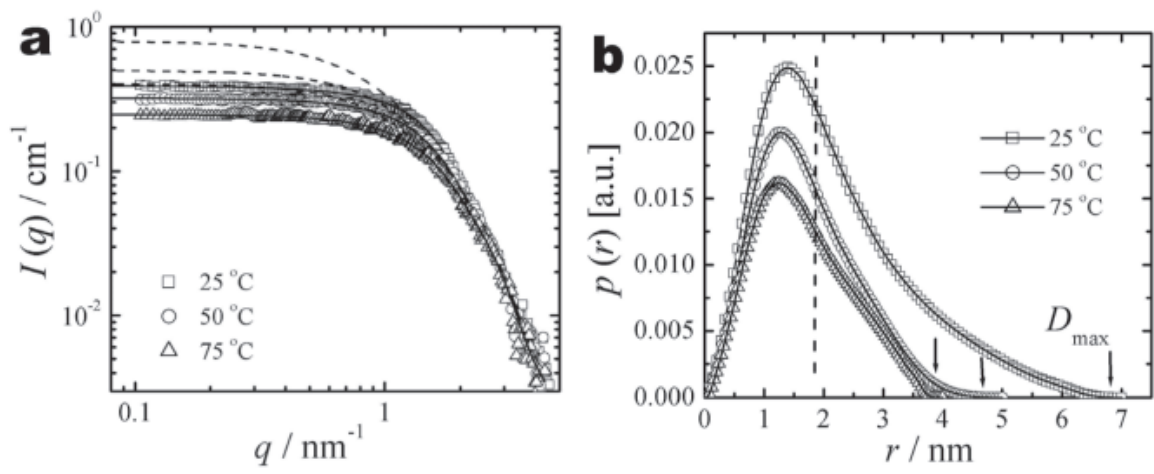

Fig. 5 Effect of Temperature on the Reverse Micellar Structures of $10 \mathrm{wt} \%$ (iso- $\left.\mathrm{C}_{18}\right)_{2} \mathrm{G}_{2} /$ HD System as Obtained by SAXS; (a) The X-ray Scattering Intensities, $I(q)$, in Absolute Unit at Different Temperatures of 25, 50, and $75^{\circ} \mathrm{C}$ (b) the Corresponding Real-space $p(r)$-Functions, Obtained by GIFT.

The solid and broken lines in panel a represent GIFT fit and the calculated form factor for $n$ particles in unit volume $n P(q)$, respectively. Arrows in panel b highlight the maximum diameter, $D_{\max }$, of the micellar core.

ing contrast profiles for the (iso- $\left.\mathrm{C}_{18}\right)_{2} \mathrm{G}_{2} / \mathrm{HD}$ system at different surfactant concentration at $25^{\circ} \mathrm{C}$. The point at which $p_{\mathrm{c}}(r)$ reaches to zero in the high- $r$ side of the $p_{\mathrm{c}}(r)$-function estimates the cross-sectional diameter. The resulting $p_{\mathrm{c}}(r)$ functions are typical of homogeneous aggregates with maximum cross-section diameter of $1.98 \mathrm{~nm}$. This value is close to the value estimated from the inflection point in total $p(r)$-function and is independent to the composition of the (iso- $\left.\mathrm{C}_{18}\right)_{2} \mathrm{G}_{2} / \mathrm{HD}$ systems. Note that the maximum core radius $\left(R_{\text {core }}\right)$ ca. to $0.99 \mathrm{~nm}$ from the contrast profile is nearly equal to twice the extended chain of the glycerol moiety, as the glycerol group alone would account for 0.4-0.5 nm.

\subsection{Temperature induced microstructural transition}

Figure 5 shows the results of SAXS measurements $(I(q)$ and $p(r)$-functions), at different temperatures $(25,50$, and $75^{\circ} \mathrm{C}$ ) for the $10 \mathrm{wt} \%\left(\text { iso- } \mathrm{C}_{18}\right)_{2} \mathrm{G}_{2} / \mathrm{HD}$ system. One can clearly see a decreasing trend of the forward scattering intensity in the low- $q$ region with the rise of temperature from 25 to $75^{\circ} \mathrm{C}$ without affecting the scattering behavior in the high- $q$ regions. Such features in the scattering intensity can be taken as an evidence of micelle shortening, which is clearer in the real space $p(r)$-functions (Fig. 5b). Increasing temperature from 25 to $75^{\circ} \mathrm{C}$, the micellar size gradually decrease from $\sim 6.70$ to $3.95 \mathrm{~nm}$ without showing a notable modification of the micellar cross-section structure, i.e., the micelles shrink by $41 \%$. 
Note that the SAXS data treatments at higher temperatures might suffer from an artifact as temperature usually worsens the contrast of the systems, and may lead to an underestimation of micellar size. However, in the reverse micelle systems, there is a positive electron density fluctuation in the hydrophilic core, whose hydrophobic shell has almost negligible contrast in the oils. This offers a good setting for the reverse systems despite the worse contrast at
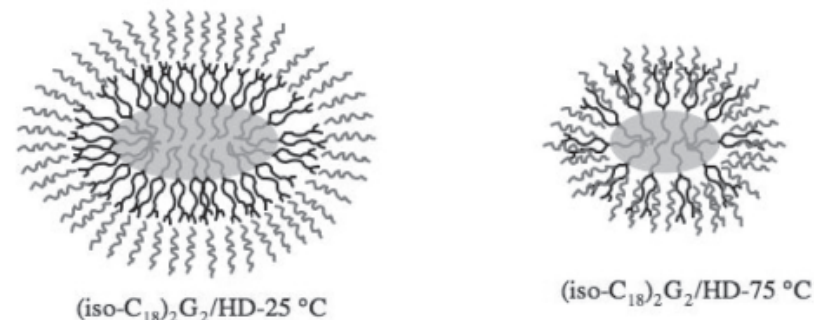

Scheme 3 Model Showing Effect of Temperature on the Reverse Micellar Structure in the $\left(\text { iso- } \mathrm{C}_{18}\right)_{2} \mathrm{G}_{2} /$ HD System. higher temperature. As shown in Fig. 5b, the decrease in the micellar size by ca. $\sim 41 \%$ with increasing temperature from 25 to $75^{\circ} \mathrm{C}$, cannot be due to the contrast problem. It seems that opposite to the poly (oxyethylene) type nonionic surfactant aqueous systems, rod-to-sphere type transition is favorable in the reverse micellar structure in nonaqueous system.

Temperature induced transition in the micellar structure can be explained in terms of enhanced penetration of HD in the surfactant chain at higher temperatures. The penetration increases the $c p p$ of the surfactant and, hence, aggregates with a more negative curvature are likely to form as shown by the model in Scheme 3. Besides, increasing temperature also increases the effective hydrophobic volume of the surfactant chain by increasing the kink states in the chain. Moreover, since the surfactant is glycerol based there could be presence of traces water as an impurity. Under this condition, increasing temperature dehydrates the surfactants head group and increases the cpp. The present result supports our previous reports, where temperature effect on the reverse micellar structure was more pronounced $^{50)}$.
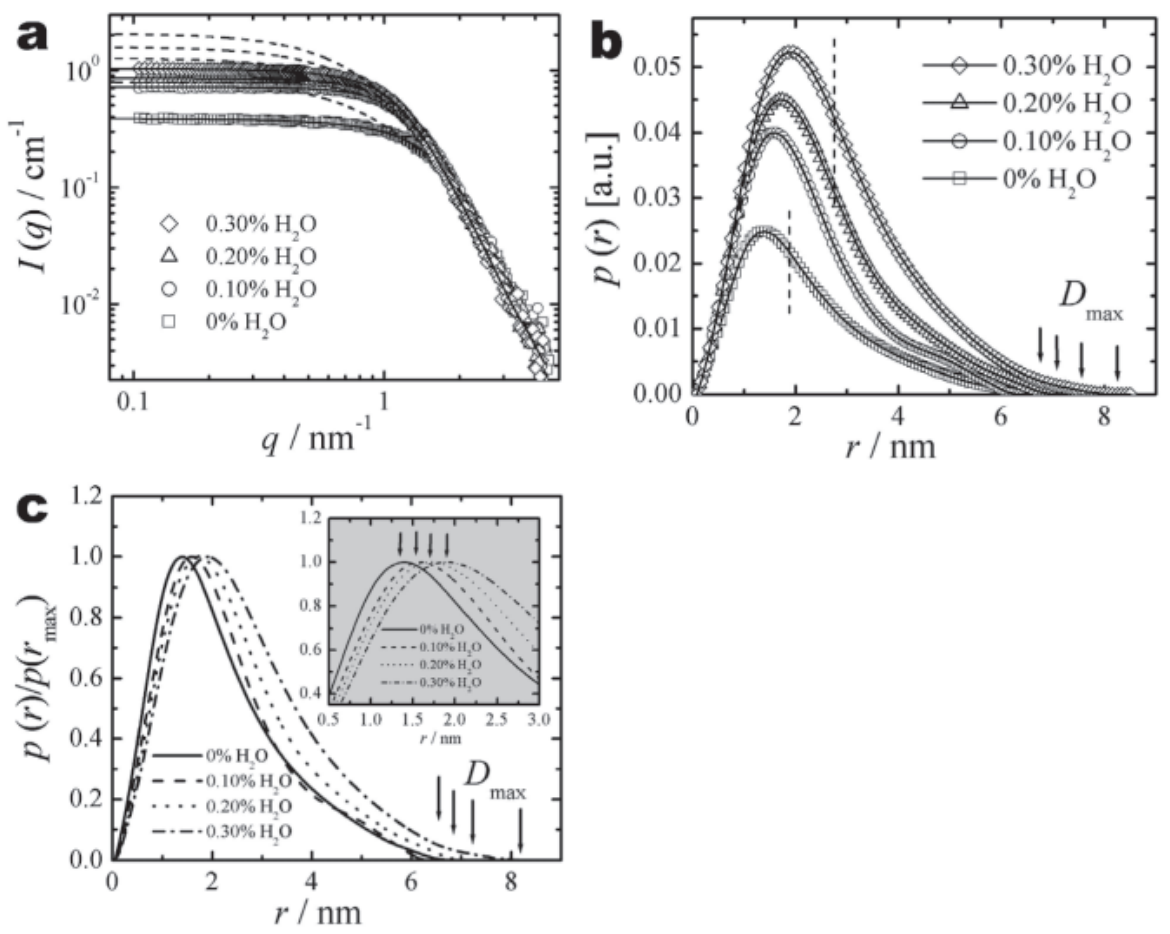

Fig. 6 (a) X-ray Scattered Intensities $I(q)$ of $10 \mathrm{wt} \%$ iso- $\mathrm{C}_{18} \mathrm{G}_{2} / \mathrm{HD}$ at Different Concentrations of Added Water in Absolute Unit at $25^{\circ} \mathrm{C}$, (b) the Corresponding Pair-distance Distribution Functions, and (c) the Normalized Pair-distance Distribution $p(r) / p\left(r_{\max }\right)$ versus $r$.

Solid and broken lines in panel a represent GIFT fit and the calculated form factor for $n$ particles in unit volume, $n P(q)$, respectively. 


\subsection{Effect of added water on the structure of host reverse micelles}

Reverse micelles have been shown to solubilize water or other polar solvents and thus act as a reservoir of various polar solvents. As a result, water-soluble drug molecules can be encapsulated inside the reverse micellar core. Structure of the reverse micelles has been found to be much bigger than the empty micelles ${ }^{51)}$. Besides, water has been shown to induce micellization in nonaqueous systems $^{52)}$.

Here we discuss the water solubilization in the reverse micelles of the $10 \mathrm{wt} \%\left(\text { iso- } \mathrm{C}_{18}\right)_{2} \mathrm{G}_{2} / \mathrm{HD}$ system at $25^{\circ} \mathrm{C}$, and the structural modulation caused by the solubilized water. It was found that the $10 \mathrm{wt} \%\left(\text { iso- } \mathrm{C}_{18}\right)_{2} \mathrm{G}_{2} / \mathrm{HD}$ system can solubilize $\sim 0.3 \%$ water. With further addition of water, phase separation was occurred. Figure 6 shows the SAXS results on the $10 \mathrm{wt} \%\left(\text { iso- } \mathrm{C}_{18}\right)_{2} \mathrm{G}_{2} / \mathrm{HD}+$ water systems at different concentrations of water.

As it can be seen in Fig. 6, addition of $0.10 \%$ water strongly enhances the forward scattering intensity, which goes on increasing with further addition of water. Such behavior in the scattering function would be best account for the micellar growth, which is better clear in the corresponding real space $p(r)$-functions shown in Fig. $6 \mathrm{~b}$. It can be seen that the maximum size of the micelles (as indicated by arrows) increases in parallel with the water concentration. Furthermore, with increasing the amount of water the position of the maximum in the $p(r)$-function shifts towards higher- $r$ side. This clearly shows that the water not only favors micellar growth, but also increases the crosssectional diameter of the micellar core. For better visibility, we present the normalized pair-distance distribution functions $p(r) / p\left(r_{\max }\right)$ in Fig. 6c, which clearly shows the movement of $r_{\max }$ position towards higher- $r$ side as the amount of water increases. Thus the present results confirmed the formation of swollen micelles with a water pool in the mi- cellar core.

Figure 7 shows the data of direct cross-section analysis for the water added systems. The core cross-section diameter ca. to $1.98 \mathrm{~nm}$ estimated from the position of $D_{\text {c max }}$ in the $p_{\mathrm{c}}(r)$-curve, and maximum radius, $R_{\text {core }}$, ca. to $0.99 \mathrm{~nm}$ from the $\Delta \rho_{\mathrm{c}}(r)$-profile for the $10 \mathrm{wt} \%\left(\text { iso- } \mathrm{C}_{18}\right)_{2} \mathrm{G}_{2} / \mathrm{HD}$ system without water addition is much smaller in comparison to the water added systems. Addition of water significantly increases the cross-section diameter of the micelles, giving $D_{\text {c max }}$ ca. to $2.90 \mathrm{~nm}$ and $R_{\text {core }}$ ca. to $1.45 \mathrm{~nm}$. Thus, the results clearly indicate the formation of water pool in the micellar core as shown by the model in Scheme 4 and emphasize an efficiency of the solubilized water for producing a thicker core of the reverse micelles.

\section{CONCLUSION}

Structure of diglycerol polyisostearate nonionic surfactant micelles in nonpolar oil $n$-hexadecane (HD) has been investigated by using a small-angle X-ray scattering tech-
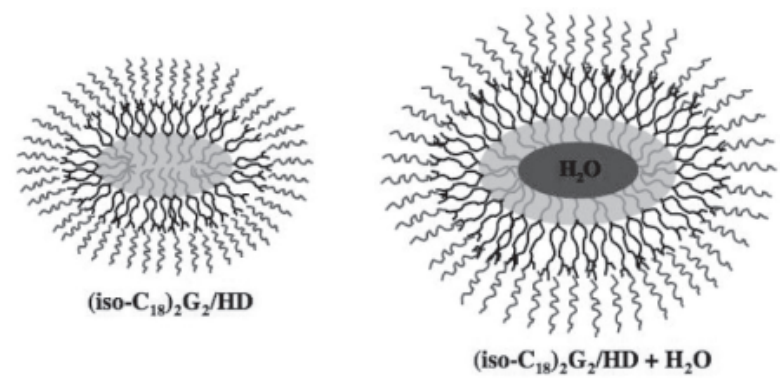

Scheme 4 Model Showing Swollen Reverse Micelles upon Addition of Water in the $10 \mathrm{wt} \%$ (iso- $\left.\mathrm{C}_{18}\right)_{2} \mathrm{G}_{2} / \mathrm{HD}$ System.
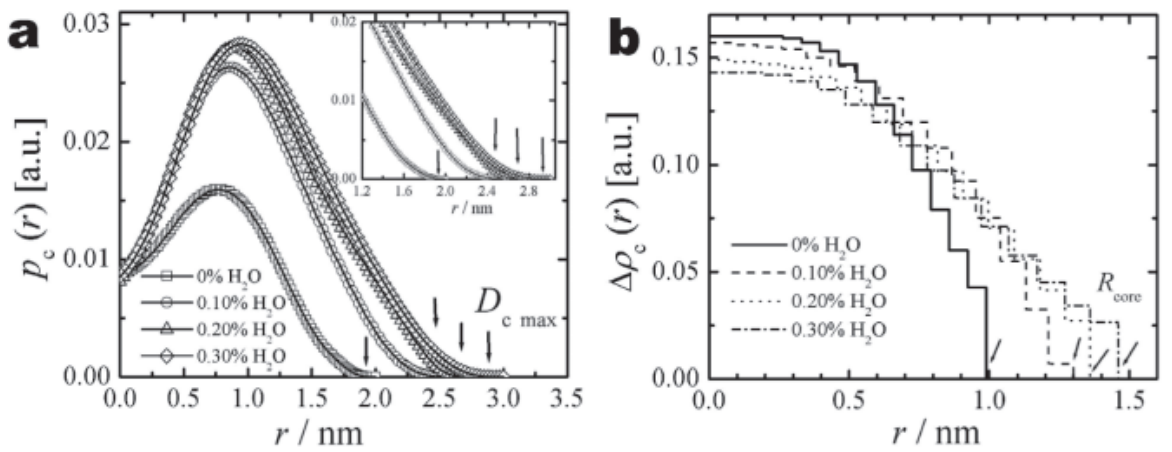

Fig. 7 (a) The Cross-sectional Pair-distance Distribution Function, $p_{\mathrm{c}}(r)$, for the 10 wt $\%$ (iso- $\left.\mathrm{C}_{18}\right)_{2} \mathrm{G}_{2} / \mathrm{HD}+$ Water Systems at Different Concentration of Water, and (b) the Corresponding Cross-section Radial Electron Density Profile, $\Delta \rho_{\mathrm{c}}(r)$, Calculated from the Deconvolution of the $p_{\mathrm{c}}(r)$. 
nique. The generalized indirect Fourier transformation (GIFT) evaluation of the scattering data and the direct model fittings have given a clear picture on the formation of variety of reverse micellar structures in the studied systems. It was found that the structure of the micelles largely depends on the lipophilic tail architecture of the surfactant. The maximum size of the aggregate goes on decreasing favoring prolate-to-sphere type transition in the micellar structure with increasing the isostearate chain per surfactant molecule. Composition could not modulate the micellar structure, but the micellar size goes on decreasing with the rise of temperature. The reverse micelles observed in the present systems efficiently solubilized some amount of water in the micellar core exploring the practical application of the studied systems. The solubilized water induced both the micellar growth and core swelling.

\section{ACKNOWLEDGEMENT}

LKS thank JSPS for Postdoctoral Fellowship for Foreign Researchers. Authors are thankful to Dr. Takaaki Sato, Shinshu University for fruitful discussion.

\section{References}

1. Luisi, P.L.; Strab, B.E. (ed.)Reverse Micelles: Biological and Technological Relevance of Amphiphilc Structures in Apolar Media. Plenum Press. New York (1987).

2. Pileni, M.P. Structure and Reactivity in Reverse Micelles. Pileni M.P.(ed.). Elasevier. Amsterdam. Vol. 65 (1989).

3. Boutonnet, M.; Kizling, J.; Stenius, P. The preparation of monodisperse colloidal metal particles from microemulsions. Colloids and Surfaces 5, 209-225 (1982).

4. López-Quintela, M.A.; Tojo, C.; Blanco, M.C.; García Rio, L.; Leis, J.R. Microemulsion dynamics and reactions in microemulsions. Current Opinion in Colloid and Interface Science 9, 264-278 (2004).

5. López-Quintela, M.A. Synthesis of nanomaterials in microemulsions: Formation mechanisms and growth control. Current Opinion in Colloid and Interface Science 8, 137-144(2003).

6. Cushing, B.L.; Kolesnichenko, V.L.; O’ Connor, C.J. Chem. Rev. 104, 3893(2004).

7. Lisiecki, I.; Pileni, M.P. Synthesis of copper metallic clusters using reverse micelles as microreactors. $J$. Am. Chem. Soc. 115, 3887-3896(1993).

8. Pileni, M.P. Nanosized particles made in colloidal assemblies. Langmuir 13, 3266-3276 (1997).

9. Sharma, S.C.; Kunieda, H.; Esquena, J.; Rodriguez-Aberu, C. Phase behavior and preparation of mesoporous silica in aqueous mixtures of fluorinated surfactant and hydrophobic fluorinated polymer. J. Colloid Interface Sci. 299, 297-304(2006).

10. Pileni, M.P. Water in oil colloidal droplets used as microreactors. Adv. Colloid Interface Sci. 46, 139-163 (1993).

11. Uddin, H.Md.; Rodriguez, C.; Watanabe, K.; LópezQuintela, M.A.; Kato, T.; Furukawa, H.; Harashima, A.; Kunieda, H. Phase behavior and formation of reverse cubic phase based emulsion in water/ poly (oxyethylene) poly (dimethylsiloxane) surfactants/ silicone oil systems. Langmuir 17, 5169-5175 (2001).

12. Kaneko, M.; Matsuzawa, K.; Uddin, H.Md.; LópezQuintela, M.A.; Kunieda, H. Effect of hydrophobic chain length of amphiphilic silicone oil (Copolymer) on the nonionic surfactant-layer curvature. J. Phys. Chem. B 108, 12736-12743(2004).

13. Kunieda, H.; Tanimoto, M.; Shigeta, K.; Rodriguez, C. Highly concentrated cubic-phase emulsions: Basic study on D-phase emulsification using isotropic gels. $J$. Oleo Sci. 50, 633-639(2001).

14. Kunieda, H.; Uddin, H.Md.; Horii, M.; Furukawa, H.; Harashima, A. Effect of hydrophilic- and hydrophobicchain lengths on the phase behavior of A - B-type silicone surfactants in water. J. Phys. Chem B 105, 5419-5426 (2001).

15. Kunieda, H.; Shigeta, K.; Ozawa, K. Suzuki, M. Self organized structures in poly (oxyethylene) oleyl etherwater systems. J. Phys. Chem. B 101, 7952-7957 (1997).

16. Forster, S.; Zisenis, M.; Wenz, E.; Antonietti, M. Micellization of strongly segregated block copolymers. J. Chem. Phys. 104, 9956-9971(1996).

17. Zhong, X.F.; Varsheny, S.K.; Eisenberg, A. Critical micelle lengths for ionic blocks in solutions of polystyrene-b-poly (sodium acrylate) ionomers. Macromolucules 25, 7160-7167 (1992).

18. Desjardins, A.; van de Ven, T.G.M.; Eisenberg, A. Colloidal properties of block ionomers. 2. Characterization of reverse micelles of styrene-b-methacrylic acid and styrene-b-metal methacrylate diblocks by dynamic light scattering. Macromolecules 25, 2412-2421 (1992).

19. Shrestha, L.K.; Kaneko, M.; Sato, T.; Acharya, D.P.; Iwanaga, T.; Kunieda, H. Phase behavior of diglycerol fatty acid esters - nonpolar oil systems. Langmuir 22, 1449-1454(2006).

20. Rodriguez, C.; Uddin H.Md.; Watanabe, K.; Furukawa, H.; Harashima, A.; Kunieda, H. Self-organization, phase behavior, and microstructures of poly (oxyethylene) poly (dimethylsiloxane) surfactants in nonpolar oil. J. Phys. Chem. B 106, 22-29(2002).

21. De, T.; Maitra, A. Solution behaviour of aerosol OT in non-polar solvents. Adv. Colloid Interface Sci. 59, 
95-193 (1995).

22. Riter, R.E.; Kimmel, J.R.; Undiks, E.P.; Levinger, N.E. Novel reverse micelles partitioning nonaqueous polar solvents in a hydrocarbon continuous phase. J. Phys. Chem. B 101, 8292-8297(1997).

23. Cason, J.P.; Roberts, C.B. Metallic copper nanoparticle synthesis in AOT reverse micelles in compressed propane and supercritical ethane solutions. J. Phys. Chem. B 104, 1217-1221 (2000).

24. Li, Q.; Li, T.; Wu, J. Comparative study on the structure of reverse micelles. 2. FT-IR, ${ }^{1} \mathrm{H}$ NMR, and electrical conductance of $\mathrm{H}_{2} \mathrm{O} / \mathrm{AOT} / \mathrm{NaDEHP} / n$-heptane systems. J. Phys. Chem. B 104, 9011-9016(2000).

25. Kanamaru, M.; Einaga, Y. Polymer-like structure of lecithin reverse micelles in the lecithin+water+cycloh exane system. Polymer 43, 3925-3936 (2002).

26. Tung, S-H.; Huang, Y-E.; Raghavan, S.R. A new reverse wormlike micellar system: Mixtures of bile salt and lecithin in organic liquids. J. Am. Chem. Soc. 128, 5751-5756 (2006)

27. Shrestha, L.K.; Sato, T.; Aramaki, K. Phase behavior of monoglycerol fatty acid esters in nonpolar oils: Reverse rodlike micelles at elevated temperatures. $J$. Phys. Chem. B 110, 12266-12273(2006).

28. Shrestha, L.K.; Sato, T.; Aramaki, K. Phase behavior and self-organized structures of diglycerol monolaurate in different nonpolar organic solvents. Langmuir 23, 6606-6613 (2007).

29. Shrestha, L.K.; Sato, T.; Aramaki, K. Shape, size, and structural control of reverse micelles in diglycerol monomyristate nonionic surfactant system. J. Phys. Chem. B. 111, 1664-1671 (2007).

30. Shrestha, L.K.; Shrestha, R.G.; Oyama, K.; Matsuzawa, M.; Aramaki, K. Structural characterization of diglycerol polyisostearate reverse micelles in organic solvents. J. Phys. Chem. B. 113, 12669-12679(2009).

31. Fritz, G.; Bergmann, A.; Glatter, O. Evaluation of small-angle scattering data of charged particles using the generalized indirect fourier transformation (GIFT) technique. J. Chem. Phys. 113, 9733-9740(2000).

32. Glatter, O.; Fritz, G.; Lindner. H.; Brunner, P.J.; Mittelbach, R.; Strey, R.; Egelhaaf, S.U. Non-ionic micelles near the critical point: micellar growth and attractive interaction. Langmuir 16, 8692-8701 (2000).

33. Brunner-Popela, J.; Mittelbach, R.; Strey, R.; Shubert, K.V.; Kaler, E.W.; Glatter, O. Small-angle scattering of interacting particles. III. D2O-C12E5 mixtures and microemulsions with $n$-octane. J. Chem. Phys. 110, 10623-10632(1999).

34. Weyerich, B.; Brunner-Popela, J.; Glatter, O. Small-angle scattering of interacting particles. II. Generalized indirect Fourier transformation under consideration of the effective structure factor for polydisperse systems. J. Appl. Crystallogr. 32, 197-209 (1999).
35. Pusey, P.N.; Fijnaut, H.M.; Vrij, A. Mode amplitudes in dynamic light scattering by concentrated liquid suspensions of polydisperse hard spheres. J. Chem. Phys. 77, 4270-4281 (1982).

36. Salgi, P.; Rajagopolan, R. Polydispersity in colloids: Implications to static structure and scattering. Adv. Colloid Interface Sci. 43, 169-288(1993).

37. Fritz, G.; Bergmann, A. Interpretation of small-angle scattering data of inhomogeneous ellipsoids. J. Appl. Cryst. 37, 815-822(2004).

38. Glatter, O. Scattering studies on colloids of biological interest (Amphiphilic systems). Progr. Colloid Polym. Sci. 84, 46-54 (1991).

39. Glatter, O. Evaluation of small-angle scattering data from lamellar and cylindrical particles by the indirect transformation method. J. Appl. Cryst. 13, 577-584 (1980).

40. Glatter, O. Determination of particle-size distribution functions from small-angle scattering data by means of the indirect transformation method. J. Appl. Crystallogr. 13, 7-11 (1980).

41. Glatter, O. Convolution square root of band-limited symmetrical functions and its application to small-angle scattering data. J. Appl. Cryst. 14, 101-108(1981).

42. Glatter, O.; Hainisch, B. Improvements in real-space deconvolution of small-angle scattering data. J. Appl. Cryst. 17, 435-441 (1984).

43. Shrestha, L.K.; Shrestha, R.G.; Varade, D.; Aramaki, K. Tunable parameters for the structural control of reverse micelles in glycerol monoisostearate/ oil systems: A SAXS study. Langmuir 25, 4435-4442 (2009).

44. Glatter, O. Computation of distance distribution functions and scattering functions of models for small-angle scattering experiments. Acta Phys. Austriaca 52, 243-256 (1980).

45. Stradner, A.; Sedgwick, H.; Cardinaux, F.; Poon, W.C.K.; Egelhaaf, S.U.; Schurtenberger, P. Equilibrium cluster formation in concentrated protein solutions and colloids. Nature 432, 492-495 (2004).

46. Stradner, A.; Glatter, O.; Schurtenberger, P. A hexanolinduced sphere-to-flexible cylinder transition in aqueous alkyl polyglucoside solutions. Langmuir 16, 5354-5364(2000).

47. Svensson, B.; Olsson, U.; Alexandridis, P.; Mortensen, K. A SANS investigation of reverse (water-in-oil) micelles of amphiphilic block copolymers. Macromolecules 32, 6725-6733 (1999).

48. Gast, A.P. Structure, interactions, and dynamics in tethered chain systems. Langmuir 12, 4060-4067 (1996).

49. Shrestha, L.K.; Glatter, O.; Aramaki, K. Structure of nonionic surfactant glycerol $\alpha$-monomyristate micelles in organic solvents: A SAXS study. J. Phys. Chem. B 113, 6290-6298(2009). 


\section{L.K. Shrestha, R.G. Shrestha, K. Oyama et al.}

50. Shrestha, L.K.; Sato, T.; Aramaki, K. Intrinsic parameters for structural variation of reverse micelles in nonionic surfactant (glycerol $\alpha$-monolaurate)/ oil systems: A SAXs study. Phys. Chem. Chem. Phys. 11, 4251-4259(2009).

51. Li, J.; Zhang, J.; Han, B.; Gao, Y.; Shen, D.; Wu, Z. Ef- fect of ionic liquid on the polarity and size of the reverse micelles in supercritical $\mathrm{CO}_{2}$. Colloid and Surfaces A 279, 208-212 (2006).

52. Mathews, M.B.; Hirschhorn, E. Solubilization and micelle formation in a hydrocarbon medium. J. Colloid Sci. 8, 86-96 (1953). 